Pacific Journal of Mathematics

MOIRE PHENOMENA IN ALGEBRAIC GEOMETRY: KeITH MILO KendIC 


\title{
MOIRÉ PHENOMENA IN ALGEBRAIC GEOMETRY: RATIONAL ALTERNATIONS IN $\boldsymbol{R}^{2}$
}

\author{
KEITH M. KeNDIG
}

This paper investigates rational alternations, principally in $R^{2}$. Rational alternations in $R^{n}$ generalize the polynomial alternations studied in the author's Moiré Phenomena in Algebraic Geometry: Polynomial Alternations in $R^{n}$. Rational alternations, like polynomial alternations, have the spirit of diffraction gratings, but may possess singularities, where grating bands flow together. Both alternations carry with them more information than ordinary varieties. As in the polynomial case, the systems of varieties making up two rational alternations generate new systems of varieties under union (or dually, intersection), corresponding to systems of moiré fringes of various orders. This paper investigates density functions naturally associated with these fringes, and studies the behavior of the fringes at points of indeterminacy of the defining functions.

1. Introduction. For the sake of motivation, we begin not with a general rational function, but with a polynomial. Thus, let $p$ be an element of a polynomial ring $k\left[X_{1}, \cdots, X_{n}\right]$ over a field $k$. Then $p$ defines an algebraic variety $V(p)=\left\{\left(x_{1}, \cdots, x_{n}\right) \mid p\left(x_{1}, \cdots, x_{n}\right)=0\right\}$ in $k^{n}=k_{x_{1}, \cdots, x_{n}}$. It is natural to ask how operations on polynomials translate into operations on the associated varieties. For instance, if $p, q \in k\left[X_{1}, \cdots, X_{n}\right]$, then

$$
V(p q)=V(p) \cup V(q) .
$$

This is especially satisfying because the variety $V(p q)$ is so easily obtained from the original pieces $V(p)$ and $V(q)$, just by taking their union. The simple from in (1.1) of course holds for ideals-that is, for ideals $a, \mathfrak{b}$ in $k\left[X_{1}, \cdots, X_{n}\right]$, we have

$$
V(\mathfrak{a} \mathfrak{b})=V(\mathfrak{a}) \cup V(\mathfrak{b}) \text {. }
$$

What about sum? That is, how is $V(p+q)$ related to $V(p)$ and $V(q) ? \quad V(p+q)$ does not have that same kind of simple geometric relationship to $V(p)$ and $V(q)$. Of course, one cannot expect a purely geometric answer to this since, for example, $V(p)=V(a p)(a \in k \backslash\{0\})$, but in general $V(p+q) \neq V(a p+q)$. Although " $V(\mathfrak{a}+\mathfrak{b})=V(\mathfrak{a}) \cap$ $V(\mathfrak{b})$ " answers an analogous ideal-theoretic question, there is still a natural polynomial question, and polynomials should not be neglected. The trouble is that since $V(p)=V(a p)(a \in k \mid\{0\})$, it seems that in taking the variety, we lose too much geometric information to hope 
to relate $V(p+q)$ with $V(p)$ and $V(q)$, and that polynomials are "really" trying to define something more informative. In this paper, we let a polynomial $p$ define a more informative geometric object " $V^{*}(p)$ ", which does not ignore multiplication by elements of the groundfield. $V^{*}(p)$ will be generalized to $V^{*}(r)(r$, a rational function) in $\S 3$. For these more informative objects, one can then reasonably hope to relate $V^{*}(p+q)$ with $V^{*}(p)$ and $V^{*}(q)$.

To keep things visual, we take $k=R$, though it appears that much of what we say can be extended to any complete valued field. We begin by noting that $V(p)$ may be regarded as the inverse image $p^{-1}(\{0\})$ of $\{0\} \in \boldsymbol{R}$. In place of $\{0\}$, we consider the set $\boldsymbol{A}=[0,1]+$ $2 \boldsymbol{Z} \subseteq \boldsymbol{R}$. Thus $\boldsymbol{A}$ consists of "half of $\boldsymbol{R}$ "-unit intervals separated by unit intervals. For $p \in \boldsymbol{R}\left[X_{1}, \cdots, X_{n}\right]$, we define $V^{*}(p)$ by

$$
V^{*}(p)=p^{-1}(\boldsymbol{A})\left(\cong \boldsymbol{R}_{x_{1}, \cdots, r_{n}}\right) .
$$

EXAMPLE 1.4.

1.4.1. $V^{*}\left(X_{1}\right) \subseteq \boldsymbol{R}_{X_{1} Y_{2}}$ is just the cylindrization along $\boldsymbol{R}_{I_{2}}$ of $\boldsymbol{A}$ and thus consists of equally-spaced bands parallel to $\boldsymbol{R}_{X_{2}}$. To a physicist, this could be considered as a "straight-line diffraction grating".

1.4.2. $V^{*}\left(X_{1}^{2}+X_{2}^{2}\right) \subseteq \boldsymbol{R}_{X_{1} X_{2}}$ consists of circular bands centered at $(0,0)$, the bounding circles having radii $\sqrt{n}(n=1,2,3, \cdots)$. $V^{*}\left(X_{1}^{2}+X_{2}^{2}\right)$ can thus be regarded as a "Fresnel diffraction plate". The bands all have equal area.

Now it is a familiar fact that superimposing diffraction rulings creates alternately light and dark fringes called "moiré fringes". Let us begin by looking at a specific example in $\boldsymbol{R}_{X_{1} X_{2}}: p=a X_{1}(a \neq 0)$, and $q=X_{1}^{2}+X_{2}^{2}$. From a purely geometric viewpoint, it is not very obvious "why" the line $V\left(a X_{1}\right)$ and the point $V\left(X_{1}^{2}+X_{2}^{2}\right)$ should yield a circle $V\left(a X_{1}+X_{1}^{2}+X_{2}^{2}\right)$ centered at $(-(a / 2), 0)$ and passing through $(0,0)$. Now look at $V^{*}\left(a X_{1}\right)$ and $V^{*}\left(X_{1}^{2}+X_{2}^{2}\right)$. In the superposition of these two diffraction rulings, there appear moiré fringes, and we see that something remarkable happens: there is a system of circular fringes centered at $(-(a / 2), 0)$ and the bands of $V^{*}\left(a X_{1}+X_{1}^{2}+X_{2}^{2}\right)$ appear to run along these fringes. In fact, $V\left(a X_{1}+X_{1}^{2}+X_{2}^{2}\right)$ is the unique "fringe curve" (cf. $\S 2)$ of this system passing through $V\left(a X_{1}\right) \cap$ $V\left(X_{1}^{2}+X_{2}^{2}\right)$. Fig. 3 in [2] shows these fringes; $a$ is approximately 9.5. This figure also shows a similar system of fringes centered at $(a / 2,0)$; this, it turns out, corresponds to subtracting $X_{1}^{2}+X_{2}^{2}$ from $a X_{1}$. If this figure were extended out further, one could even detect fainter systems further out, which correspond to adding (or sudtracting) various integral multiples of $X_{1}^{2}+X_{2}^{2}$ from $a X_{1}$. 
REMARK 1.5. Both $V(p)$ and $V^{*}(p)$ have strengths and weaknesses, and these tend to complement each other. For instance, at the ground field level, we have:

$V$ is good at distinguishing $V(p)$ from $V(a+p)$, but

$V$ is poor at distinguishing $V(p)$ from $V(a p)(a \in \boldsymbol{R} \backslash\{0\})$.

$V^{*}$ is good at distinguishing $V^{*}(p)$ from $V^{*}(a p)$

$\left(V^{*}(a p)\right.$ is in obvious sense "finer" than $V^{*}(p)$ if $|a| \supsetneqq 1$, and "coarser" if $|a| \varsubsetneqq 1$, but

$V^{*}$ is less good at distinguishing $V^{*}(p)$ from $V^{*}(a+p)$

$\left(V^{*}(a+p)=V^{*}\left(a^{\prime}+p\right)\right.$ if $\left.a-a^{\prime} \in 2 Z\right)$.

At the "nonconstant polynomial level" we have, for relatively prime polynomials $p, q \in \boldsymbol{R}\left[X_{1}, X_{2}\right] \backslash \boldsymbol{R}$ :

$V^{*}$ is good at expressing $V^{*}(p+q)$ in terms of $V^{*}(p)$ and $V^{*}(q)$, but

$V^{*}$ is poor at expressing $V^{*}(p q)$ in terms of $V^{*}(p)$ and $V^{*}(q)$.

$V$ is good at expressing $V(p q)$ in terms of $V(q)$, but

$V$ is poor at expressing $V(p+q)$ in terms of $V(p)$ and $V(q)$.

Both $V$ and $V^{*}$ have equal claim to producing varieties from ideals, in the sense that

$$
V(\mathfrak{a})=\underset{p \in \mathfrak{a}}{V}(p)=\underset{p \in \mathfrak{a}}{*}(p) \stackrel{\text { def }}{=} V^{*}(\mathfrak{a}) .
$$

This whole idea of moiré fringes arising from superimposing diffraction gratings has been recognized and used in various physical sciences for some time. In 1926, for instance, Ronchi ([4]) succeeded in using the moiré effect to test lens systems for aberrations. Until the early 1950's, further progress was somewhat slow in coming due to the difficulty in creating sufficiently fine gratings. After that, there followed a great many applications of the moiré effect for very precise measurement. (See [3] and [5] for bibliographies.) More recently, some exciting applications of the moiré effect have been found. As one example, moiré fringes have been observed in electron micrographs of thin, crossed crystals. Such fringes supply far more information and detail than available from ordinary electron micrographs of single crystals. For instance, crystal dislocations of less than the diameter of one atom ( $\cong 1$ angstrom) can be detected, which represents a resolving power of some 100 to 1000 times greater than that of the electron microscope itself.

Relative to the question raised at the beginning of this paper, 
we have, in rough terms, the following: Given relatively prime polynomials $p, q \in \boldsymbol{R}\left[X_{1}, \cdots, X_{n}\right] \backslash \boldsymbol{R}, V^{*}(p) \cup V^{*}(q)$ displays different systems of fringes, some obvious and others, fainter and not so obvious. These fringes correspond to rational linear combinations of $p$ and $q$; the fringes alternate between light and dark-in fact, the density "tries" to oscillate about $3 / 4$ in a piecewise linear way. The amplitudes of these oscillations are "arithmetic in character"-that is, in a linear combination $c_{1} p+c_{2} q$, if $c_{2} / c_{1}$ is rational, then the limit of the amplitudes as $b \rightarrow \infty$ depends on the prime factors of $c_{2} / c_{1}$. If the prime factors are large and odd, this limit is small; if $c_{2} / c_{1}$ is irrational or a prime factor is even, this limit is zero.

These ideas have been worked out in [2]; in $\S 2$ next, we briefly summarize what we need of [2] in the present paper.

Everything we have mentioned so far deals with polynomials. However, one can go further and consider $V^{*}(r)=r^{-1}(A)$, where $\boldsymbol{r}=\boldsymbol{r}\left(X_{1}, \cdots, X_{n}\right) \in \boldsymbol{R}\left(X_{1}, \cdots, X_{n}\right) \backslash \boldsymbol{R}$ is a rational function. Such "diffraction rulings" now may possess "singular points", where the bands flow together. This is in contrast to $V^{*}(p)$, where all the bands are disjoint.

This is not solely of mathematical interest; it arises in physical applications, just as the varieties $V^{*}(p)$ do. For example, this behavior is approximated in stress-strain problems when the material under consideration is subjected at certain points to very high stress or strain; stress-strain lines flow together at such points. We will see that points where bands of $V^{*}(r)$ flow together are points of indeterminacy of the rational function $r$.

The present paper is devoted to extending [2] to the rational case. This happens also to extend the affine study in [2] to a projective study, since polynomials, when recentered at a point at infinity, become no worse than rational functions.

\section{Some basic results in the polynomial case.}

Definition 2.1. The subset $\boldsymbol{A}=[0,1]+2 \boldsymbol{Z}$ of $\boldsymbol{R}$ is called the basic linear alternation on $\boldsymbol{R}$.

Definition 2.2. Let $p \in \boldsymbol{R}\left[X_{1}, X_{2}\right] \backslash \boldsymbol{R}$. Then $p^{-1}(\boldsymbol{A}) \subseteq \boldsymbol{R}_{X_{1} X_{2}}$ is called a polynomial alternation in $\boldsymbol{R}_{X_{1} X_{2}}$, and is denoted by $V^{*}(p)$.

Let $p, q \in \boldsymbol{R}\left[X_{1}, X_{2}\right] \backslash \boldsymbol{R}$, with $(p, q)=1$. For any nonzero linear combination $c_{1} p+c_{2} q\left(c_{i} \in \boldsymbol{R}\right)$, we shall look for fringes in $V^{*}(p) \cup V^{*}(q)$ running along the level varieties defined by $c_{1} p+c_{2} q$. Because it will simplify the exposition somewhat, in the rest of this paper we write $m$ in place of $-\left(c_{2} / c_{1}\right)$. (The letter $m$ reminds us of "slope".) Thus we will consider the varieties $q=m p+b(b \in \boldsymbol{R}, m \in \boldsymbol{R} \cup\{\infty\})$. We 
make the convention that $m=\infty$ in $q=m p+b$ means $p+b=0$.

Since we will be testing for density along real curves defined by $q=m p+b$, we need the following definition.

DeFinition 2.3. Let $p \in \boldsymbol{R}\left[X_{1}, X_{2}\right] \backslash \boldsymbol{R}$. Then $V(p) \subseteq \boldsymbol{R}_{X_{1} X_{2}}$ consists of infinitely many points, or only finitely many points, or else the set is $\varnothing . V(p)$ has dimension 1,0 or -1 , respectively. In the first $V(p)$ is called a real curve in $\boldsymbol{R}_{X_{1} X_{2}}$, and we sometimes use the suggestive letter $C$ for $V(p)$. If $P$ is not isolated in $C$, we say that $C$ has dimension 1 at $P$, and write $\operatorname{dim}_{P} C=1$. Similarly, if $P$ is isolated in $C$, we write $\operatorname{dim}_{P} C=0$; if $P \notin C$, $\operatorname{dim}_{P} C=-1$.

REMARK 2.4. Let $p, q \in \boldsymbol{R}\left[X_{1}, X_{2}\right] \backslash \boldsymbol{R}$ with $(p, q)=1$; let $b \in \boldsymbol{R}$, and let $m$ be fixed in $\boldsymbol{R} \cup\{\infty\}$. Then the variety $V(q-m p-b)$, which we denote by $V_{b}(q-m p)$, is a curve either for all $b \in \boldsymbol{R}$, or for all $b$ in a half line of $\boldsymbol{R}$. (See [2], Lemma 5.6.) When we use a phrase like "the curve $V_{b}(q-m p)$ ", we shall be assuming that $V_{b}(q-m p)$ is a real curve. As $b$ runs through $\boldsymbol{R}$ in the first case, the collection of curves clearly covers $R_{X_{1} X_{2}}$. As $b$ runs through the half line in the second case, it is easy to check that the collection of these curves covers all points of $\boldsymbol{R}_{X_{1} X_{2}}$ except possibly on some real curve of $\boldsymbol{R}_{X_{1} X_{2}}$.

Let $\left\{V_{b}\right\}$ be the collection of real curves chosen from $V_{b}(q-m p)$, as $b$ runs through $\boldsymbol{R}$. For fixed $m,\left\{V_{b}\right\}$ reveals " $m$ th order moiré behavior" if the density of $V^{*}(p) \cup V^{*}(q)$ within the curves of $\left\{V_{b}\right\}$ oscillates in $b$. (We still need to define "density".) We refer to these testing curves as mth order (moiré) fringes, or simply (moiré) fringes; the collection of these curves is called the $m$ th order fringe family.

DEFinition 2.5. Let $S$ be a countable union of intervals in $\boldsymbol{R}$. Then $S$ has density $\mathfrak{D}$ in $R$ if for any $\varepsilon>0$, there is a decomposition of $\boldsymbol{R}$ into a disjoint union of intervals $T_{i} \subseteq \boldsymbol{R}$, which are uniformly bounded in length, so that

$$
\left|\mathfrak{D}-\frac{\text { length }\left(S \cap T_{i}\right)}{\text { length } T_{i}}\right|<\varepsilon
$$

for all but possibly finitely many $T_{i}$.

Notation 2.6. $\Delta(X): \boldsymbol{R} \rightarrow \boldsymbol{R}$ denotes the periodic "triangle function" defined in the interval of periodicity $[-1,1)$ by $\Delta(X)=$ $1-2|X|$.

If $p$ and $q$ are linear, we have the following basic result. (See [2], $\$ 3$ for the proof.) 
TheOREM 2.7. Let $\left(X_{1}, X_{2}\right)=X$, let $p(X)=a \cdot X$, and let $q(X)=$ $a^{\prime} \cdot X$, where $a$ and $a^{\prime}$ are linearly independent in $\boldsymbol{R}_{X}$. Then any line in $\boldsymbol{R}_{X}$ can be written as

$$
L_{b}: q=m p+b
$$

for some $m \in \boldsymbol{R} \cup\{\infty\}$ and $b \in \boldsymbol{R}$. Let $\mathfrak{D}\left(L_{b}\right)$ denote the density of $\left[V^{*}(p) \cup V^{*}(q)\right] \cap L_{b}$ in $L_{b}$. Then:

$$
\begin{aligned}
& \left\{\begin{array}{ll}
\mathfrak{D}\left(L_{b}\right)=1 & (b \in \boldsymbol{A}) \\
\mathfrak{D}\left(L_{b}\right)=\frac{1}{2} & (b \notin \boldsymbol{A})
\end{array}\right\} \text { if } m=0 \text { or } \infty ; \\
& \left\{\mathfrak{D}\left(L_{b}\right)=\frac{3}{4}-\frac{\Delta\left(n_{1} b\right)}{4 n_{1} n_{2}}\right\} \\
& \text { if } m=\frac{n_{1}}{n_{2}} \in \boldsymbol{Q} \backslash\{0\}, \text { where }\left(n_{1}, n_{2}\right)=1 \text {, and } n_{1} n_{2} \text { is odd. }
\end{aligned}
$$

$$
\mathfrak{D}\left(L_{b}\right)=\frac{3}{4} \text { for all other } m .
$$

The $L_{b}$ in (2.7.2) singles out those directions in which $V^{*}(p) \cup V^{*}(q)$ displays moire behavior; in these directions the density oscillates about $3 / 4$ in a piecewise linear way. The amplitude is small when at least one of $n_{1}, n_{2}$ is large. (2.7.3) includes the limiting case in which the fraction becomes irrational $\left(n_{1}, n_{2} \rightarrow \infty\right)$, the oscillation about $3 / 4$ then becoming zero.

In place of the above linear polynomials $p$ and $q$, we now consider arbitrary relatively prime $p, q \in \boldsymbol{R}\left[X_{1}, X_{2}\right] \backslash \boldsymbol{R}$. The following notion of "refinement" essentially makes the underlying rulings $V^{*}(p)$ and $V^{*}(q)$ very fine relative to the fringe bands in $V^{*}(p) \cup V^{*}(q)$ and one can then approximate each of the curves $q=m p+b$ by a piecewise linear curve to get a more general version of Theorem 2.7.

Definition 2.8. Let $p, q \in \boldsymbol{R}\left[X_{1}, X_{2}\right] \backslash \boldsymbol{R}$, where $(p, q)=1$. For any positive integer $k$ and any $m \in \boldsymbol{R} \cup\{\infty\}$, define $\left(s_{m}, t_{m}\right)$ by

$$
\left\{\begin{array}{lll}
(k p, m k p+(q-m p)) & \text { if } \quad m \in \boldsymbol{R} \\
(p, k q) & \text { if } \quad m=\infty .
\end{array}\right.
$$

Then $V^{*}\left(s_{m}\right) \cup V^{*}\left(t_{m}\right)$ is called the k-refinement of $V^{*}(p) \cup V^{*}(q)$ along the $m$ th order fringe family.

Definition 2.9. Let $p, q \in \boldsymbol{R}\left[X_{1}, X_{2}\right] \backslash \boldsymbol{R}$, where $(p, q)=1$; let $P \in$ $\boldsymbol{R}_{X_{1} X_{2}}$, and let $m \in \boldsymbol{R} \cup\{\infty\}$. Then $V^{*}(p) \cup V^{*}(q)$ has limit density $D_{P}$ at $P$ with respect to $m$ if the following holds: For every $\varepsilon>0$ there 
is a $\delta>0$ such that for all positive $\rho$ less than $\delta$, there is a $k_{\rho}>0$ so that for all $k>k_{\rho}$,

$$
\left|\grave{D}_{P}-\frac{\operatorname{area}\left[\left(V^{*}\left(s_{m}\right) \cup V^{*}\left(t_{m}\right)\right) \cap B(P, \rho)\right]}{\operatorname{area}[B(P, \rho)]}\right|<\varepsilon .
$$

Here $B(P, \rho)$ denotes the open disk of radius $\rho$, centered at $P$.

One can show that for fixed $m \in \boldsymbol{R} \cup\{\infty\}$, the above $\supset_{P}$ is constant along any fringe curve $V_{b}(q-m p)$. With this $\grave{D}_{P}$, Theorem 2.5 then holds for arbitrary relatively prime polynomials $p, q \in \boldsymbol{R}\left[X_{1}, X_{2}\right] \backslash \boldsymbol{R}$. See [2], §6 for details and proofs.

3. Some qualitative results about rational alternations and moirés.

We now begin our generalization to the "rational" level. We start with some preliminary definitions and notations.

Definition 3.1. For any $r \in \boldsymbol{R}\left(X_{1}, X_{2}\right) \backslash \boldsymbol{R}, \boldsymbol{r}^{-1}(\boldsymbol{A})\left(\subseteq \boldsymbol{R}_{X_{1} X_{2}}\right)$ is called a rational alternation on $\boldsymbol{R}_{X_{1} X_{2}}$; we denote it by $V^{*}(r)$.

Notation 3.2. For any $r \in \boldsymbol{R}\left(X_{1}, X_{2}\right) \backslash \boldsymbol{R}$, write $r=p / q$, where $(p, q)=1$. For $b \in \boldsymbol{R}$, let $V_{b}(r)$ denote $V(p-b q)$; let $V_{\infty}(r)$ denote $V(q)$. If $r$ is clear from context, we may write simply $V_{b}$ or $V_{\infty}$. In each case the variety may be a curve, consist of only finitely many points, or be $\varnothing$. If in a particular situation we wish to assume that any of these is actually a curve, we will use a phrase like "the curve $V_{b}^{\prime \prime}$. Note that $V_{0}(r)$ and $V_{\infty}(r)$ share no one-dimensional components. Let $r_{1}, r_{2} \in \boldsymbol{R}\left(X_{1}, X_{2}\right) \backslash \boldsymbol{R}$, and write for $i=1,2, r_{i}=p_{i} / q_{i}$, where $\left(p_{i}, q_{i}\right)=1$. Assume that $\left(r_{1}, r_{2}\right)=1$-that is, that no two of $p_{1}, p_{2}$, $q_{1}, q_{2}$ share a nonconstant factor, and let us agree that $V_{b}\left(r_{2}-m r_{1}\right)$ means $V_{b}\left(r_{1}\right)$ when $m=\infty$. Then for fixed $m \in \boldsymbol{R} \cup\{\infty\}$ and $b \in \boldsymbol{R} \cup$ $\{\infty\}$, any curve $V_{b}\left(r_{2}-m r_{1}\right)$ is called an $m$ th order (moiré) fringe, or simply a (moiré) fringe. One can then define the k-refinement of $V^{*}\left(r_{1}\right) \cup V^{*}\left(r_{2}\right)$ along the $m$ th order fringe family just as in Definition 2.8, replacing $p$ and $q$ there by $r_{1}$ and $r_{2}$, respectively. Also, at any $P \in \boldsymbol{R}_{X_{1} X_{2}} \backslash\left(V_{\infty}\left(r_{2}-m r_{1}\right)\right)$, one can similarly generalize $\grave{D}_{P}$ in Definition 2.9 by replacing $p$ and $q$ there by $r_{1}$ and $r_{2}$. One can then show, exactly as in the polynomial case (see [2], $\S 6$ ), that $\searrow_{P}$ is constant on each $V_{b}\left(r_{2}-m r_{1}\right) \backslash V_{\infty}\left(r_{2}-m r_{1}\right)(b \in \boldsymbol{R})$, and one can define $\mathfrak{D}\left(V_{b}\left(r_{2}-m r_{1}\right)\right)$ to be this constant value. With these definitions, the proof in [2], $\S 6$ of the polynomial version of Theorem 2.5, extends essentially verbatim to the $r_{1}, r_{2}$-level.

We know at this stage that for $r_{1}, r_{2}$ as above, any two varieties $V_{b_{1}}\left(r_{2}-m r_{1}\right)$ and $V_{b_{2}}\left(r_{2}-m r_{1}\right)\left(b_{1} \neq b_{2}\right)$ are disjoint in $\boldsymbol{R}_{X_{1} X_{2}} \backslash V_{\infty}(r)$, and 
we have determined the density induced on curves $V_{b}\left(r_{2}-m r_{1}\right)$ $(b \in \boldsymbol{R})$ by $V^{*}\left(r_{1}\right) \cup V^{*}\left(r_{2}\right)$. To extend our results to all of $\boldsymbol{R}_{X_{1} X_{2}}$, we next study the behavior of curves $V_{b}\left(r_{2}-m r_{1}\right)$ near points of $V_{\infty}\left(r_{2}-m r_{1}\right)$. The remainder of this paper is devoted to this study. We work out some basic qualitative results in this section. In $\S 4$ we find a density function.

We begin this study by determining, for a given $r \in R\left(X_{1}, X_{2}\right) \backslash \boldsymbol{R}$, the "fixed points" in the family $\left\{V_{b}(r) \mid b \in \boldsymbol{R} \cup\{\infty\}\right\}$. First, since any two different $V_{b_{1}}(r), V_{b_{2}}(r)$ are disjoint in $R_{X_{1} x_{2}} \backslash V_{\infty}(r)$, we know $V_{b_{1}}(r) \cap$ $V_{b_{2}}(r) \subseteq V_{\infty}(r)$.

Lemma 3.3. Let $r \in \boldsymbol{R}\left(X_{1}, X_{2}\right) \backslash \boldsymbol{R}$, and let $P$ be a point of $\boldsymbol{R}_{X_{1} X_{2}}$. If there exist $b_{1}, b_{2} \in \boldsymbol{R} \cup\{\infty\}\left(b_{1} \neq b_{2}\right)$ such that $P \in V_{b_{1}}(r) \cap V_{b_{2}}(r)$, then $P$ is contained in every $V_{b}(r)(b \in \boldsymbol{R} \cup\{\infty\})$.

Notation 3.4. The set of all $P$ satisfying the conclusion in Lemma 3.3 is denoted by $W(r)$, or by simply $W$ if $r$ is clear from context.

REMARK 3.5. If $r$ is a polynomial, then $W(r)=\varnothing$.

Proof of Lemma 3.3. Write $r=p_{1} / p_{2}, p_{i} \in R\left[X_{1}, X_{2}\right],\left(p_{1}, p_{2}\right)=1$, and rewrite $p_{1} / p_{2}=b_{i}$ as $b_{i 1} p_{1}+b_{i 2} p_{2}=0\left(b_{i j} \in R\right)$, where $\left\{b_{i 1}=0, b_{i 2} \neq 0\right\}$ corresponds to $b_{i}=0,\left\{b_{i 1} \neq 0, b_{i 2}=0\right\}$ corresponds to $b_{i}=\infty$, and $\left\{b_{i 1} \neq 0, b_{i 2} \neq 0\right\}$ corresponds to $b_{i} \in R \backslash\{0\}$. Then the condition $P \in$ $V_{b_{1}}(r) \cap V_{b_{2}}(r)$ in the lemma becomes

$$
\begin{aligned}
& b_{11} p_{1}(P)+b_{12} p_{2}(P)=0, \\
& b_{21} p_{1}(P)+b_{22} p_{2}(P)=0 .
\end{aligned}
$$

Now $b_{1} \neq b_{2}$ implies that (3.6) has rank two; therefore $p_{1}(P)=p_{2}(P)=$ 0 . Thus for any $b=c_{1} / c_{2} \in R \cup\{\infty\}$, we have $c_{1} p_{1}(P)-c_{2} p_{2}(P)=0$-that is, $P \in V_{b}(r)$.

Here is an example of Lemma 3.3.

EXAMPLE 3.7. Let $r=\left(X_{1}^{2}+X_{2}^{2}\right) / X_{1} X_{2}$. Then for all $|b| \varsubsetneqq 2, V_{b}=$ $\{(0,0)\} \subseteq \boldsymbol{R}_{X_{1} X_{2}} . \quad V_{2}(r)$ is the line $X_{1}-X_{2}=0$, and $V_{-2}(r)$ is the line $X_{1}+X_{2}=0$. For $|b| \supsetneqq 2, V_{b}$ splits up into two lines, one approaching $\boldsymbol{R}_{X_{1}}$ and the other approaching $\boldsymbol{R}_{X_{2}}$, as $b \rightarrow \infty . V_{\infty}=\boldsymbol{R}_{X_{1}} \cup \boldsymbol{R}_{X_{2}}$. Note that Lemma 3.3 holds $\left(P\right.$ is $(0,0)$ ), even though $V_{b}$ is not always a curve.

The next result relates $V_{\infty}\left(r_{2}-m r_{1}\right)$ with $V_{\infty}\left(r_{1}\right)$ and $V_{\infty}\left(r_{2}\right)$.

LEMMA 3.8.

For any $m \in R \backslash\{0\}, V_{\infty}\left(r_{2}-m r_{1}\right) \subseteq V_{\infty}\left(r_{1}\right) \cup V_{\infty}\left(r_{2}\right)$. 
If $r_{i}=p_{i} / q_{i}$, where $\left(p_{i}, q_{i}\right)=1$, and if $\left(q_{1}, q_{2}\right)=1$, then equality holds in (3.8.1).

Proof. $\subseteq$ : Obvious.

$=$ : Follows at once from the following lemma:

Lemma 3.9. Let $p_{i}, q_{i} \in \boldsymbol{R}\left[X_{1}, X_{1}\right]$, where $\left(p_{1}, q_{1}\right)=\left(p_{2}, q_{2}\right)=1$, and let $m \in \boldsymbol{R} \backslash\{0\}$. If $p_{2} q_{1}-m p_{1} q_{2} \neq 0$, then

$$
\left(p_{2} q_{1}-m p_{1} q_{2}, q_{1} q_{2}\right)=1 \Longleftrightarrow\left(q_{1}, q_{2}\right)=1 \text {. }
$$

Proof. $\Rightarrow$ : Suppose that $\left(q_{1}, q_{2}\right)=s \in R\left[X_{1}, X_{2}\right] \backslash R$. Then $s$ is a factor of $q_{1} q_{2}$, and also a factor of $p_{2} q_{1}-m p_{1} p_{2}$.

$\Longleftarrow:$ Suppose $\left(q_{1}, q_{2}\right)=1$. Let $t$ be any prime factor of $q_{1} q_{2}$. We assume without loss of generality that $t \mid q_{1}$. Then $t p_{2} q_{1}$. Therefore $t \mid p_{2} q_{1}-m p_{1} q_{2}$ iff $t \mid p_{1} q_{2}$. However $\left(p_{1}, q_{1}\right)=1$ and $\left(q_{1}, q_{2}\right)=1$ imply, respectively, that $t \nmid p_{1}$ and $t \nmid q_{2}$. Thus $t \nmid p_{1} q_{2}$, and therefore $t \nmid p_{2} q_{1}-$ $m p_{1} q_{2}$. Thus $\left(p_{2} q_{1}-m p_{1} q_{2}, q_{1} q_{2}\right)=1$, which proves " $\Longleftarrow$ ".

The following ties in the "fixed points" of a moiré with those of the underlying alternations.

Lemma 3.'10. Let $m \in \boldsymbol{R} \backslash\{0\}$, and let $r_{1}=p_{1} / q_{1}, r_{2}=p_{2} / q_{2}$ and $r_{2}-m r_{1}$ be elements of $\boldsymbol{R}\left(X_{1}, X_{2}\right) \backslash \boldsymbol{R}$. If $\left(q_{1}, q_{2}\right)=1$, then

$$
W\left(r_{2}-m r_{1}\right)=W\left(r_{1}\right) \cup W\left(r_{2}\right) \cup\left(V_{\infty}(r) \cap V_{\infty}\left(r_{2}\right)\right) \text {. }
$$

Proof. First, $r_{2}-m r_{1}=\left(p, q_{1}-m p_{1} q_{2}\right) / q_{1} q_{2}$. Now $W\left(r_{2}-m r_{1}\right)$ consists of those points of $\boldsymbol{R}_{X_{1} X_{2}}$ where $q_{1} q_{2}$ and $p_{2} q_{1}-m p_{1} q_{2}$ are simultaneously zero. If both $q_{1}$ and $q_{2}$ vanish at $P \in W\left(r_{2}-m r_{1}\right)$, then $P \in V_{\infty}\left(r_{1}\right) \cap V_{\infty}\left(r_{2}\right)$. If $q_{1}(P)=0$ but $q_{2}(P) \neq 0$, then $p_{2} q_{1}-m p_{1} q_{2}=0$ implies that $p_{1}(P)=0$, so $P \in W\left(r_{1}\right)$. Similarly, if $q_{2}(P)=0$ and $q_{1}(P) \neq 0$, then $P \in W\left(r_{2}\right)$.

REMARK 3.11. If $q_{1}$ and $q_{2}$ are not relatively prime, then (3.10.1) may not hold. For example if $r_{1}=\left(X_{1}+1\right) / X_{2}, r_{2}=\left(X_{1}-1\right) / X_{2}$, then $W\left(r_{1}+r_{2}\right)=\{(0,0)\}$, while $(3.10 .1)$ in this case is $\boldsymbol{R}_{X_{1}}$.

Moiré fringes, as well as the underlying alternations, display a wide range of behavior at points of $W$. For example let $r_{1}=X_{1}$, and consider the projective completion in $\boldsymbol{P}^{2}(\boldsymbol{R})$ of $V^{*}\left(r_{1}\right) \subseteq \boldsymbol{R}_{X_{1} X_{2}}$. The boundaries of the bands all pass through a single, common point on the line at infinity, and any two such boundaries intersect there transversally. If $r_{2}=X_{2}$, then the same holds for the boundaries of the completion of $V^{*}\left(r_{2}\right)$ at a different point at infinity; similarly for all the curves $V_{b}\left(X_{2}-m X_{1}\right)$ (for any fixed $m \in \boldsymbol{R} \cup\{\infty\}$ ). However, 
it may happen that each of the underlying alternations of a moiré has the above kind of transversally intersecting boundaries at infinity, but that for some $m$, the $m$ th order moiré fringes may fail to have this property. For example, the boundaries of $V^{*}\left(X_{1} / X_{2}\right)$ intersect transversally at $(0,0)$; likewise for $\left.V^{*}\left(X_{1}+X_{1}^{2} / X_{2}\right)\right)$. However, any two fringes corresponding to $m=1$ approach $(0,0)$ in a mutually tangent manner. (We give definitions in a moment.) Just the opposite can happen, too. For example, any two boundaries of $V^{*}\left(X_{1} / X_{2}^{2}\right)$ are tangent at $(0,0)$; similarly for the boundaries of $\left.V^{*}\left(X_{1}+X_{1}^{2}\right) / X_{2}^{2}\right)$. However, any two fringes corresponding to $m=1$ intersect transversally at $(0,0)$. These are only two of a number of cases.

There exists at nine-way classification which covers all such possibilities. For this, we will need a few preliminary definitions and results.

First, throughout the rest of this section $P$ will denote an arbitrary, fixed point in $\boldsymbol{R}_{X_{1} X_{2}}$. We will use the notion of germ at $P$ of a real curve $C$. If $P \notin C$ or if $P$ is isolated in $C$ (i.e., if $\operatorname{dim}_{P} C=-1$ or 0 ), the usual germ can be identified with $\varnothing$ and $P$, respectively. However for our purposes, it will simplify the exposition to agree that the notion germ of $C$ at $P$ carries with it the assumption that $\operatorname{dim}_{P} C=1$. Since $P$ is fixed throughout, if $\operatorname{dim}_{P} C=1$ then the germ of $C$ at $P$ will be denoted by $C^{\sim}$.

We next consider the notion of tangent cone to a germ at $P$; this generalizes "tangent space" at a point of a differentiable manifold. This will be useful when considering transversality and tangency.

Definition 3.12. Suppose $C^{\sim}$ exists, and let $C$ be a representative of $C^{\sim}$ in a $\boldsymbol{R}_{X_{1} X_{2}}$-open neighborhood of $P$. The tangent cone to $C^{\sim}$, denoted by $T\left(C^{\sim}\right)$, is $\{P\}$ together with the set of all vectors $v$ based at $P$ satisfying this condition: there is a sequence of points $\left\{P_{i}\right\}, P_{i} \in C \backslash P, P_{i} \rightarrow P$, and a sequence of numbers $\left\{r_{i}\right\}$ such that $v=$ $\lim _{i \rightarrow \infty} r_{i}\left(P_{i}-P\right)$.

ExAmple 3.13. Suppose $P=(0,0) \in \boldsymbol{R}_{X_{1} X_{2}}$. For $C \cong \boldsymbol{R}_{X_{1} X_{2}}$ defined by $X_{2}^{2}-X_{1}^{3}, T\left(C^{\sim}\right)=\boldsymbol{R}_{X_{1}}$; for $C \cong \boldsymbol{R}_{X_{1} X_{2}}$ defined by $X_{2}^{2}-X_{1}\left(X_{1}^{2}-1\right)$, $T\left(C^{\sim}\right)$ consists of the two lines $X_{1}+X_{2}=0$ and $X_{1}-X_{2}=0$.

The following algebraic characterization of $T\left(C^{\sim}\right)$ is important in the sequel.

THEOREM 3.14. Let $C \cong \boldsymbol{R}_{X_{1} X_{2}}$ be defined by a polynomial $p \in$ $\boldsymbol{R}\left[X_{1}, X_{2}\right] \backslash \boldsymbol{R}$, let $\operatorname{dim}_{P} C=1$, and let $p^{\dagger}$ denote the initial part of $p$ is expanded about $P$. Then $T\left(C^{\sim}\right)$ is the zero set in $\boldsymbol{R}_{X_{1} X_{2}}$ of $p^{\dagger}$.

Proof. Theorem 3.14 is a special case of Theorem 2.8 in [1] 
(which, in fact, includes the case when $\operatorname{dim}_{P} C=-1$ or 0 , though we don't need this here).

REMARK 3.15. $T\left(C^{\sim}\right)$ in Theorem 3.13 consists of finitely many lines through $P$. Thus both $T\left(C^{\sim}\right)$ and $p^{\dagger}$ are homogeneous with respect to $P$. If $p$ has no nonconstant repeated factors, and if the Jacobian $\left(p_{X_{1}}, p_{X_{2}}\right)$ has rank one at $P$, then the zero set of $p$ in $C_{X_{1} X_{2}}$ is a real differentiable manifold in a $\boldsymbol{C}^{2}$-neighborhood of $P, C \leqq \boldsymbol{R}_{X_{1} X_{2}}$ is smooth at $P$, and $T\left(C^{\sim}\right)$ coincides with the usual (real) tangent line to $C$ at $P$.

EXAMPLE 3.16. Let $P=(0,0) \in \boldsymbol{R}_{X_{1} X_{2}}$. For $C \cong \boldsymbol{R}_{X_{1} X_{2}}$ defined by $X_{2}^{3}-X_{1}^{3}, T\left(C^{\sim}\right)$ is defined by $X_{2}^{2}=0$ - that is, $T\left(C^{\sim}\right)=\boldsymbol{R}_{X_{1}}$; for $C$ defined by $X_{2}^{2}-X_{1}\left(X_{1}^{2}-1\right), T\left(C^{\sim}\right)$ is defined by $X_{2}^{2}-X_{1}^{2}=0$ - that is, it consists of the two lines $X_{1}+X_{2}=0$ and $X_{1}-X_{2}=0$. (Cf. Example 3.12.)

Definition 3.17. Let $P \in \boldsymbol{R}_{X_{1} X_{2}}$, and suppose curves $C_{1}$ and $C_{2}$ in $\boldsymbol{R}_{X_{1} X_{2}}$ both have dimension one at $P$. Then $C_{1}^{\sim}$ and $C_{2}^{\sim}$ are said to be tangent along (precisely) $T\left(C_{1}^{\sim}\right) \cap T\left(C_{2}^{\sim}\right)$. If $T\left(C_{1}^{\sim}\right)=T\left(C_{2}^{\sim}\right)$, we say $C_{1}^{\sim}$ and $C_{2}^{\sim}$ are fully tangent. If $T\left(C_{1}^{\sim}\right) \cap T\left(C_{2}^{\sim}\right)=P$, we say that $C_{1}^{\sim}$ and $C_{2}^{\sim}$ intersect transversally.

REMARK 3.18. Let the curves $C_{i}$ in Definition 3.16 be defined by $p_{i} \in \boldsymbol{R}\left[X_{1}, X_{2}\right] \backslash \boldsymbol{R}$. Then $C_{1}^{\sim}$ and $C_{2}^{\sim}$ intersect transversally iff $p_{1}^{\sim}$ and $p_{2}^{+}$have no common real linear factors.

We now look at the behavior at points of $W_{\infty}$ of a given rational alternation $V^{*}(r)\left(r \in \boldsymbol{R}\left(X_{1}, X_{2}\right) \backslash \boldsymbol{R}\right)$. Write $r=p / q$, where $(p, q)=1$.

THEOREM 3.19. With $p, q$ and $r$ as above, let $P$ be a fixed, arbitrary point in $W(r)$, and let $p^{\dagger}$ and $q^{\dagger}$ be the initial forms of $p$ and $q$ at $P$.

3.19.1. If $\operatorname{deg} p^{\dagger}=\operatorname{deg} q^{\dagger}$, then for all but at most one value $\boldsymbol{b}_{0}$ of $b \in \boldsymbol{R}$, any two $V_{\widehat{b}_{1}}^{\sim}(r), V_{b_{2}}^{\sim}(r)\left(b_{1} \neq b_{2}, b_{1}, b_{2} \in \boldsymbol{R}\right)$ are tangent at $P$ along precisely $V\left(p^{\dagger}, q^{\dagger}\right)$. If there is a $b_{0}$ satisfying $p^{\dagger}=b_{0} q^{\dagger}$, then $V_{b_{0}}^{\sim}(r)$, if it exists, is tangent at $P$ to every other $V_{b}^{\sim}(r)$ iff each real linear factor of $\left(p^{\dagger}, q^{\dagger}\right)$ is a factor of $\left(p-b_{0} q\right)^{\dagger}$.

3.19.2. If $\operatorname{deg} p^{\dagger} \supseteqq \operatorname{deg} q^{\dagger}$, then any two $V_{b_{1}}^{\sim}(r), V_{b_{2}}^{\sim}(r)\left(b_{1} \neq b_{2}, b_{1}\right.$, $\left.b_{2} \in \boldsymbol{R}\right)$ are fully tangent, with common tangent cone $V\left(p^{\dagger}\right) . \quad V_{\infty}^{\sim}(r)$ is tangent to $V_{b}^{\sim}(\boldsymbol{r})(b \in \boldsymbol{R})$ along precisely $V\left(p^{\dagger}, q^{\dagger}\right)$. 
3.19.3. If $\operatorname{deg} p^{\dagger} \supsetneqq \operatorname{deg} q^{\dagger}$, then any two $V_{b_{1}}^{\sim}(r), V_{b_{2}}^{\widetilde{r}}(r)\left(b_{1} \neq b_{2}, b_{1}\right.$, $\left.b_{2} \in(\boldsymbol{R} \backslash\{0\}) \cup\{\infty\}\right)$ are fully tangent, the common tangent cone being $V\left(q^{\dagger}\right) . \quad V_{0}^{\sim}(r)$ is tangent to $V_{b}^{\sim}(r)(b \in(\boldsymbol{R} \backslash\{0\}) \cup\{\infty\})$ along precisely $V\left(p^{\dagger}, q^{\dagger}\right)$.

\section{Proof.}

3.19.1. If $p^{\dagger} \neq b_{i} q^{\dagger}$, then $\left(p-b_{i} q\right)^{\dagger}=p^{\dagger}-b_{i} q^{\dagger}(i=1,2)$. Then 3.19 .1 follows at once from this fact:

Suppose $s$ and $t$ are homogeneous polynomials in $R\left[X_{1}, X_{2}\right] \backslash \boldsymbol{R}$ having the same degree, and let $b_{1}, b_{2}\left(b_{1} \neq b_{2}\right)$ be two arbitrary elements of $\boldsymbol{R}$. If $s+b_{1} t$ and $s+b_{2} t$ are nonzero, then (up to a nonzero constant factor) $(s, t)=\left(s+b_{1} t, s+b_{2} t\right)$.

We prove this as follows: First, clearly $(s, t) \mid\left(s+b_{1} t, s+b_{2} t\right)$. We wish to show that $\left(s+b_{1} t, s+b_{2} t\right) \mid(s, t)$. For this, suppose $u \mid\left(s+b_{1} t\right.$, $\left.s+b_{2} t\right)$ - say

$$
\left.\begin{array}{l}
s+b_{1} t=u v_{1} \\
s+b_{2} t=u v_{2}
\end{array}\right\} \quad\left(u, v_{i} \in R\left[X_{1}, X_{2}\right]\right)
$$

Solving these two equations for $s$ and $t$ gives

$$
\begin{aligned}
& s=u\left(v_{1}-b\left(v_{2}-v_{1}\right) / b_{2}-b_{1}\right) \\
& t=u\left(v_{2}-v_{1}\right) / b_{2}-b_{1} .
\end{aligned}
$$

Thus $u \mid\left(s_{1} t\right)$, so $\left(s+b_{1} t, s+b_{2} t\right) \mid\left(s_{1} t\right)$.

3.19.2. For any $b \in \boldsymbol{R}$ we have $(p-b q)^{\dagger}=p^{\dagger}$, and the first sentence of 3.19.2 follows. Now when $b=\infty$, instead of $p-b q$ we may write $a_{1} p-a_{2} q$, where $a_{1}=0$ and $a_{2} \neq 0$; this gives $(p-b q)^{\dagger}=$ $q^{\dagger}$. Since $(p-b q)^{\dagger}=p^{\dagger}$ for any other $b$, the second sentence of 3.18.2 follows.

3.19.3. For any $b \in(\boldsymbol{R} \backslash\{0\}) \cup\{\infty\}$, we have $(p-b q)^{\dagger}=q^{\dagger}$, giving the first sentence of 3.19.3. When $b=0,(p-b q)^{\dagger}=p^{\dagger}$, and the second sentence follows.

We next turn our attention to the question of how at a point $P$, the geometric behavior of rational alternations $r_{1}^{-1}(A), r_{2}^{-1}(A) \subseteq$ $\boldsymbol{R}_{X_{1} X_{2}}$ influences the geometry at $P$ of the $m$ th-order fringes of $r_{1}^{-1}(\boldsymbol{A}) \cup$ $r_{2}^{-1}(A)$. The next theorem is the promised $3 \times 3$ classification of local behavior of moiré fringes in the neighborhood $P$.

Before stating it, suppose that $r_{i}=p_{i} / q_{i}$, where $\left(p_{i}, q_{i}\right)=1$ $(i=1,2)$; now if, for example, $p_{1}=p_{2}$ and $q_{1}=q_{2}$, then $V^{*}\left(r_{1}\right) \cup V^{*}\left(r_{2}\right)=$ 
$V^{*}\left(r_{1}\right)=V^{*}\left(r_{2}\right)$. The resulting moiré fringes $V_{b}\left(r_{2}-m r_{1}\right)=$ $V_{b}\left(\left(p_{2} q_{1}-m p_{1} q_{2}\right) / q_{1} q_{2}\right)$ are in a sense "trivial". However by Lemma 3.9 , it is clear that assuming $\left(q_{1}, q_{2}\right)=1$ avoids this behavior. We make this assumption in Theorem 3.20 , next.

THEOREM 3.20. Let $r_{1}=p_{1} / q_{1}, r_{2}=p_{2} / q_{2}$, where $r_{i} \in \boldsymbol{R}\left(X_{1}, X_{2}\right) \backslash \boldsymbol{R}$. Let $p_{i}^{\dagger}, q_{i}^{\dagger}$ be the respective parts at $P$ of $p_{i}, q_{i}(i=1,2)$, and assume that $\left(p_{1}, q_{1}\right)=\left(p_{2}, q_{2}\right)=\left(q_{1}, q_{2}\right)=1$. We then have the following classification where, for any $m \in R \backslash\{0\}, V_{b}^{\sim}$ denotes $V_{b}^{\sim}\left(r_{2}-m r_{1}\right)$, and where the italisized statement under $(i, j)(i=\mathrm{I}, \mathrm{II}, \mathrm{III} ; j=1,2,3)$ is the conclusion obtained from assuming $i$ and $j$.

I. $\operatorname{ord}_{P}\left(p_{1} / q_{1}\right)=\operatorname{ord}_{P}\left(p_{2} / q_{2}\right)$

1. $\operatorname{ord}_{P}\left(p_{1}\right)=\operatorname{ord}_{P}\left(q_{1}\right)$

(a) If $p_{2}^{\dagger} q_{1}^{\dagger}-\mathrm{m} p_{1}^{\dagger} q_{2}^{\dagger} \neq 0$, then any two different germs $V_{b_{1}}^{\sim}$ and $V_{b_{2}}$ $\left(b_{1}, b_{2} \in \boldsymbol{R} \cup\{\infty\}\right)$ are tangent along precisely $V\left(p_{2}^{\dagger} q_{1}^{\dagger}-m p_{1}^{\dagger} q_{2}^{\dagger}, q_{1}^{\dagger} q_{2}^{\dagger}\right)$.

(b) If $p_{2}^{\dagger} q_{1}^{\dagger}-m p_{1}^{\dagger} q_{2}^{\dagger}=0$, then any two germs $V_{b_{1}}^{\sim}$ and $V_{b_{2}}^{\sim}\left(b_{1}, b_{2} \in\right.$ $(\boldsymbol{R} \backslash\{0\}) \cup\{\infty\})$ are fully tangent, the tangent cone being $V\left(q_{1}^{\dagger}\right) \cup V\left(q_{2}^{\dagger}\right)$; and $V_{0}^{\sim}$ is tangent to each $V_{b}^{\sim}(b \in(\boldsymbol{R} \backslash\{0\}) \cup\{\infty\})$ along precisely $V\left(p_{2}^{\dagger} q_{1}^{\dagger}-m p_{1}^{\dagger} q_{2}^{\dagger}, q_{1}^{\dagger} q_{2}^{\dagger}\right)$.

2. $\operatorname{ord}_{P}\left(p_{1}\right) \varsubsetneqq \operatorname{ord}_{P}\left(q_{1}\right)$

(a) If $p_{2}^{\dagger} q_{1}^{\dagger}-m p_{1}^{\dagger} q_{2}^{\dagger} \neq 0$, then any two germs $V_{b_{1}}^{\sim}$ and $V_{b_{2}}^{\sim}\left(b_{1}, b_{2} \in \boldsymbol{R}\right)$ are fully tangent, the tangent cone being $V\left(p_{2}^{\dagger} q_{1}^{\dagger}-m p_{1}^{\dagger} q_{2}^{\dagger}\right)$. $V_{\infty}^{\sim}$ is tangent to each $V_{b}^{\sim}(b \in R)$ along precisely $V\left(p_{2}^{\dagger} q_{1}^{\dagger}-m p_{1}^{\dagger} q_{2}^{\dagger}, q_{1}^{\dagger} q_{2}^{\dagger}\right)$.

(b) If $p_{2}^{\dagger} q_{1}^{\dagger}-m p_{1}^{\dagger} q_{2}^{\dagger}=0$, then any of $>$, =, or $<$ may hold between $\operatorname{deg}\left(\left(p_{2} q_{1}-m p_{1} q_{2}\right)^{\dagger}\right)$ and $\operatorname{deg}\left(\left(q_{1} q_{2}\right)^{\dagger}\right)$, respectively. For " $>$ ", conclusion (I, 1(b)) holds; for " =", conclusion (I, 1(a)) holds; for " $<$ ", conclusion (I, 2(a)) holds.

3. $\operatorname{ord}_{P}\left(p_{1}\right) \supsetneqq \operatorname{ord}_{P}\left(q_{1}\right)$

Conclusion is same as that of (I, 1(b)).

II. $\operatorname{ord}_{P}\left(p_{1} / q_{1}\right) \varsubsetneqq \operatorname{ord}_{P}\left(p_{2} / q_{2}\right)$

1. $\operatorname{ord}_{P}\left(p_{1}\right)=\operatorname{ord}_{P}\left(q_{1}\right)$

Any two different germs $V_{b_{1}}^{\sim}$ and $V_{b_{2}}^{\sim}\left(b_{1}, b_{2} \in R \cup\{\infty\}\right)$ are tangent along precisely $V\left(q_{2}^{\dagger}\right) \cup V\left(p_{1}^{\dagger}, q_{1}^{\dagger}\right)$.

2. $\operatorname{ord}_{P}\left(p_{1}\right) \supseteqq \operatorname{ord}_{P}\left(q_{1}\right)$

Any two germs $V_{b_{1}}$ and $V_{b_{2}}\left(b_{1}, b_{2} \in R\right)$ are fully tangent, the tangent cone being $V\left(p_{1}^{\dagger}\right) \cup V\left(q_{2}^{\dagger}\right)$. $\quad V_{\infty}^{\sim}$ is in tangent to each $V_{b}^{\sim}(b \in \boldsymbol{R})$ along precisely $V\left(q_{2}^{\dagger}\right) \cup V\left(p_{1}^{\dagger}, q_{1}^{\dagger}\right)$.

3. $\operatorname{ord}_{P}\left(p_{1}\right) \supsetneqq \operatorname{ord}_{P}\left(q_{1}\right)$

Any two germs $V_{b_{1}}^{\sim}$ and $V_{b_{2}}^{\sim}\left(b_{1}, b_{2} \in(R \backslash\{0\}) \cup\{\infty\}\right)$ are fully tangent, with tangent cone $V\left(q_{1}^{\dagger}\right) \cup V\left(q_{2}^{\dagger}\right) . \quad V_{0}^{\sim}$ is tangent to each $V_{b}^{\sim}$ $(b \in(\boldsymbol{R} \backslash\{0\}) \cup\{\infty\})$ along precisely $V\left(q_{2}^{+}\right) \cup V\left(p_{1}^{\dagger}, q_{1}^{\dagger}\right)$. 
III. $\quad \operatorname{ord}_{P}\left(p_{1} / q_{1}\right) \supsetneqq \operatorname{ord}_{P}\left(p_{2} / q_{2}\right)$

Same as in II, except that subscripts " 1 " and " 2 " of $p$ and $q$ are everywhere reversed.

REMARK 3.21. Among the polynomials $p_{i}, q_{i}(i=1,2)$ satisfying the condition stated at the beginning of Theorem 3.20 , almost all of them also satisfy

$$
\left(p_{1}^{\dagger}, q_{1}^{\dagger}\right)=\left(p_{2}^{\dagger}, q_{2}^{\dagger}\right)=\left(q_{1}^{\dagger}, q_{2}^{\dagger}\right)=1 .
$$

More precisely, for a fixed positive integer $d$, there is in the vector space $Y_{1}$ (resp. $Y_{2}, Z_{1}, Z_{2}$ ) of polynomials $p_{1}$ (resp. $p_{2}, q_{1}, q_{2}$ ) of degree $\leqq d$, a proper algebraic subvariety $A_{1}$ (resp. $A_{2}, B_{1}, B_{2}$ ) such that for polynomials $p_{1} \in Y_{1} \backslash A_{1}, p_{2} \in Y_{2} \backslash A_{2}, q_{1} \in Z_{1} \backslash B_{1}$ and $q_{2} \in Z_{2} \backslash B_{2}, 3.21 .1$ holds. In this sense, the conclusion of the following corollary of Theorem 3.20 "almost always" holds.

CoROLlaRy 3.22. If in Theorem 3.20, 3.21.1 holds, then in Theorem 3.20 , any variety written in the form $V($,$) , is P$. In each such

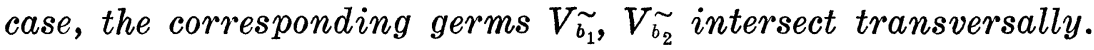

Proof of Corollary 3.22. Cases II and III are clear, since $\left(p_{1}^{\dagger}, q_{1}^{\dagger}\right)=$ 1. For case $I$, it suffices to show that $\left(p_{2}^{\dagger} q_{1}^{\dagger}-m p_{1}^{\dagger} q_{2}^{\dagger}, q_{1}^{\dagger} q_{2}^{\dagger}\right)=1$. But this follows at once from Lemma 3.9 , with $p_{i}, q_{i}$ replaced by $p_{i}^{\dagger}, q_{i}^{\dagger}$ $(i=1,2)$.

Proof of Theorem 3.20. In each case we compare $\operatorname{deg}\left(\left(p_{2} q_{1}-\right.\right.$ $\left.\left.m p_{1} q_{2}\right)^{\dagger}\right)$ with $\operatorname{deg}\left(\left(q_{1} q_{2}\right)^{\dagger}\right)$ and apply Theorem 3.19.

I. Note that $\operatorname{ord}_{P}\left(p_{1} / q_{1}\right)=\operatorname{ord}_{P}\left(p_{2} / q_{2}\right)$ implies that $\operatorname{ord}_{P}\left(p_{1} q_{2}\right)=$ $\operatorname{ord}_{P}\left(p_{2} q_{1}\right)$. Now

$$
p_{2}^{\dagger} q_{1}^{\dagger}-m p_{1}^{\dagger} q_{2}^{\dagger} \neq 0 \Longrightarrow\left(p_{2} q_{1}-m p_{1} q_{2}\right)^{\dagger}=p_{2}^{\dagger} q_{1}^{\dagger}-m p_{1}^{\dagger} q_{2}^{\dagger} ;
$$

and

$$
p_{2}^{\dagger} q_{1}^{\dagger}-m p_{1}^{\dagger} q_{2}^{\dagger}=0 \Longrightarrow \operatorname{deg}\left(\left(p_{2} q_{1}-m_{1} p_{2}\right)^{\dagger}\right) \supsetneqq \operatorname{deg} p_{1}^{\dagger} q_{2}^{\dagger}
$$

$\left(=\operatorname{deg} p_{2}^{\dagger} q_{1}^{\dagger}\right)$.

1. $\operatorname{ord}_{P}\left(p_{1} / q_{1}\right)=\operatorname{ord}_{P}\left(p_{2} / q_{2}\right)$ and $\operatorname{ord}_{P}\left(p_{1}\right)=\operatorname{ord}_{P}\left(q_{1}\right)$ imply that $\operatorname{ord}_{P}\left(p_{2}\right)=\operatorname{ord}_{P}\left(q_{2}\right)$. Thus in this case deg $p_{i}^{\dagger}=\operatorname{deg} q_{i}^{\dagger}(i=1,2)$. Therefore if $p_{2}^{\dagger} q_{1}^{\dagger}-m p_{1}^{\dagger} q_{2}^{\dagger} \neq 0$, then $\operatorname{deg}\left(p_{2}^{\dagger} q_{1}^{\dagger}-m p_{1}^{\dagger} q_{2}^{\dagger}=\operatorname{deg} q_{1}^{\dagger} q_{2}^{\dagger}\right.$. This, together with 3.23 and Theorem 3.19 .1 establishes (I, 1(a)). Next, suppose that $p_{2}^{\dagger} q_{1}^{\dagger}-m p_{1}^{\dagger} q_{2}^{\dagger}=0$. Then $\operatorname{deg}\left(\left(p_{2} q_{1}-m p_{1} q_{2}\right)^{\dagger}\right) \supsetneqq \operatorname{deg} q_{1}^{\dagger} q_{2}^{\dagger}$; this together with Theorem 3.18.3 establishes (I, 1(b)).

2(a). $\operatorname{ord}_{P}\left(p_{1} / q_{1}\right)=\operatorname{ord}_{P}\left(p_{2} / q_{2}\right)$ together with $\operatorname{ord}_{P}\left(p_{1}\right) \supsetneqq \operatorname{ord}_{P}\left(q_{1}\right)$ imply that $\operatorname{ord}_{P}\left(p_{2}\right) \varsubsetneqq \operatorname{ord}_{P}\left(q_{2}\right) . \quad p_{2}^{+} q_{1}^{+}-m p_{1}^{+} q_{2}^{+} \neq 0$ then implies that 
$\operatorname{deg}\left(p_{2}^{\dagger} q_{1}^{\dagger}-m p_{1}^{\dagger} q_{2}^{\dagger}\right) \lessgtr \operatorname{deg} q_{1}^{\dagger} q_{2}^{\dagger}$. This, with 3.23 and Theorem 3.19.2 proves $(\mathrm{I}, 2(\mathrm{a}))$. ( $\mathrm{I}, 2(\mathrm{~b}))$ is clear.

3. $\operatorname{ord}_{P}\left(p_{1} / q_{1}\right)=\operatorname{ord}_{P}\left(p_{2} / q_{2}\right)$ together with $\operatorname{ord}_{P}\left(p_{1}\right) \supsetneqq \operatorname{ord}_{P}\left(q_{1}\right)$ imply that $\operatorname{deg}\left(p_{2}^{\dagger} q_{1}^{\dagger}-m p_{1}^{\dagger} q_{2}^{\dagger}\right) \supsetneqq \operatorname{deg}\left(q_{1}^{\dagger} q_{2}^{\dagger}\right)$; if $p_{2}^{\dagger} q_{1}^{\dagger}-m p_{1}^{\dagger} q_{2}^{\dagger} \neq 0$, then 3.23 implies

$$
\operatorname{deg}\left(\left(p_{2} q_{1}-m p_{1} q_{2}\right)^{\dagger}\right) \supsetneqq \operatorname{deg}\left(q_{1}^{\dagger} q_{2}^{\dagger}\right) .
$$

If $p_{2}^{\dagger} q_{1}^{\dagger}-m p_{1}^{\dagger} q_{2}^{\dagger}=0$, then (3.24) shows that (3.25) holds a fortiori. Now applying Theorem 3.19.3 establishes (I, 3).

II. Note that $\operatorname{ord}_{P}\left(p_{1} / q_{1}\right) \varsubsetneqq \operatorname{ord}_{P}\left(p_{2} / q_{2}\right)$ implies that $\operatorname{ord}_{P}\left(p_{1} q_{2}\right) \varsubsetneqq$ $\operatorname{ord}_{P}\left(p_{,} q_{1}\right)$; hence in this case

$$
\left(p_{2} q_{1}-m p_{1} q_{2}\right)^{\dagger}=-m p_{2}^{\dagger} q_{1}^{\dagger} .
$$

1. $\operatorname{ord}_{P}\left(p_{1}\right)=\operatorname{ord}_{P}\left(q_{1}\right)$ (that is, deg $p_{1}^{\dagger}=\operatorname{deg} q_{1}^{\dagger}$ ) and (3.26) imply that $\operatorname{deg}\left(\left(p_{2} q_{1}-m p_{1} q_{2}\right)^{\dagger}\right)=\operatorname{deg}\left(q_{1}^{\dagger} q_{2}^{\dagger}\right)$. Theorem 3.19.1 now establishes (II, 1).

2. $\operatorname{ord}_{P}\left(p_{1}\right) \varsubsetneqq \operatorname{ord}_{P}\left(q_{1}\right)$ and (3.26) show that $\operatorname{deg}\left(\left(p_{2} q_{1}-m p_{1} q_{2}\right)^{\dagger}\right) \varsubsetneqq$ $\operatorname{deg}\left(q_{1}^{\dagger} q_{2}^{\dagger}\right)$. Then Theorem 3.19.2 establishes (II, 2).

3. In anology to the above, we have $\operatorname{deg}\left(\left(p_{2} q_{1}-m p_{1} q_{2}\right)^{\dagger} \supsetneqq\right.$ $\operatorname{deg}\left(q_{1}^{\dagger} q_{1}^{\dagger}\right)$, and Theorem 3.19.3 establishes (II, 3).

III. The proofs are the same as those in II, except that the subscripts " 1 " and " 2 " of $p$ and $q$ are everywhere reversed.

Here is an example of Theorem 3.20.

EXAMPLE 3.23. Let $\varepsilon>0$, and in the notation of Theorem 3.20 let $r_{1}=p_{1} / q_{1}=1 /(Y+\varepsilon X), r_{2}=p_{2} / q_{2}=1 /(Y-\varepsilon X)$. We consider fringes in $V^{*}(1 /(Y+\varepsilon X)) \cup V^{*}(1 /(Y-\varepsilon X))$, corresponding to $m=1$, passing through singular points - that is, through points of $W_{2}\left(r_{2}-m r_{1}\right)$. Now from Lemma 3.10 we see that $W\left(r_{2}-m r_{1}\right)=W\left(r_{1}\right) \cup W\left(r_{2}\right) \cup$ $\left(V_{\infty}\left(r_{1}\right) \cap V_{\infty}\left(r_{2}\right)\right)$. In this example, any two different $V_{b}\left(r_{1}\right)$ are disjoint (they are the mutually parallel lines $Y+\varepsilon X=1 / b)$; similarly for $V_{b}\left(r_{2}\right)$. Hence $W\left(r_{1}\right)=W\left(r_{2}\right)=\varnothing$. But $V_{\infty}\left(r_{1}\right)=V(Y+\varepsilon X)$ and $V_{\infty}\left(r_{2}\right)=V(Y-\varepsilon X)$, so $V_{\infty}\left(r_{1}\right) \cap m_{\infty}\left(r_{2}\right)=\{(0,0)\}$. Hence $W\left(r_{2}-m r_{1}\right)$ consists of the single point $W\left(r_{1}\right) \cup W\left(r_{2}\right) \cup\left(V_{\infty}\left(r_{1}\right) \cap V_{\infty}\left(r_{2}\right)\right)=\{(0,0)\}-$ that is, each 1 -fringe passes through $(0,0)$. This can be seen in Figure 1, where the obvious fringes correspond to $m=1$. $V_{b}\left(r_{2}-m r_{1}\right)$ is given by $1 /(Y+\varepsilon X)-1 /(Y-\varepsilon X)=b$, which reduces to

$$
\frac{\left(X-\frac{1}{\varepsilon b}\right)^{2}}{\left(\frac{1}{\varepsilon b}\right)^{2}}-\frac{Y^{2}}{\left(\frac{1}{b}\right)^{2}}=1 \text {. }
$$




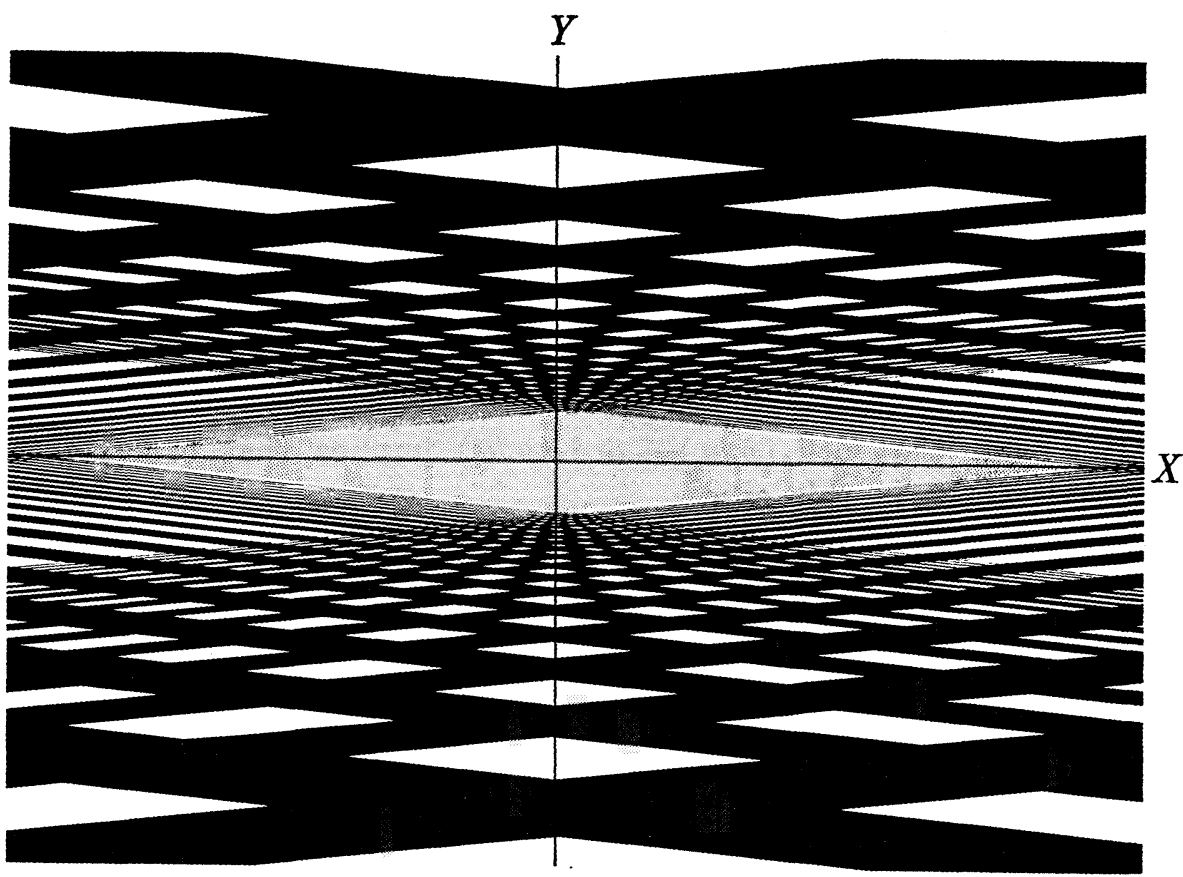

FIGURE 1

For each $b \in \boldsymbol{R}$, one branch of the above hyperbola passes through $(0,0)$.

Let $P \doteq(0,0)$. Then $\operatorname{ord}_{P}\left(p_{1} / q_{1}\right)=\operatorname{ord}_{P}\left(p_{2} / q_{2}\right)=-1$. We see that (I, 2(a)) of Theorem 3.20 applies, since also $\operatorname{ord}_{P}\left(p_{1}\right)=0 \leqq \operatorname{ord}_{P}\left(q_{1}\right)=$ 1 , and $p_{2}^{+} q_{1}^{\dagger}-m p_{1}^{\dagger} q_{2}^{\dagger}=1 \cdot(Y+\varepsilon X)-1 \cdot(Y-\varepsilon X)=2 \varepsilon X \neq 0$. The theorem then tells us that any two germs $V_{b_{1}}^{\sim}\left(r_{2}-m r_{1}\right)$ and $V_{b_{2}}\left(r_{2}-m r_{1}\right)$ $\left(b_{1}, b_{2} \in R\right)$ are fully tangent, the tangent cone being $V(2 \varepsilon X)=\boldsymbol{R}_{Y}$. This is also evident in Fig. 1. For $b=\infty,(\mathrm{I}, 2(\mathrm{a}))$ tells us that $V_{\infty}^{\sim}\left(r_{2}-m r_{1}\right)$ is tangent to each $V_{b}^{\sim}\left(r_{2}-m r_{1}\right)(b \in R)$ along $V\left(p_{2}^{\dagger} q_{1}^{\dagger}-\right.$ $\left.m p_{1}^{\dagger} q_{2}^{\dagger}, q_{1}^{\dagger} q_{2}^{\dagger}\right)=V(2 \varepsilon X,(Y+\varepsilon X)(Y-\varepsilon X))=\{(0,0)\}$ - that is, that $V_{\infty}^{\sim}\left(r_{2}-m r_{1}\right)$ is transverse to each $V_{b}^{\sim}\left(r_{2}-m r_{1}\right)(b \in R)$ at $(0,0)$. This is in fact so, since a representative of $V_{\infty}^{\sim}\left(r_{2}-m r_{1}\right)$ is $\boldsymbol{R}_{X}$, which is transverse to $\boldsymbol{R}_{Y}$ at $(0,0)$.

Note. The fringes running along the other branches of the hyperbola in (3.23.1) cross $R_{x}$ at $1 / \varepsilon b$. These fringes are especially obvious only for smaller values of $b$-that is, when $1 / \varepsilon b$ is largeand therefore do not appear in Figure 1.

Incidentally, one can also verify Lemma 3.8 in this case, too. $V_{\infty}\left(r_{1}\right)=V(Y+\varepsilon X)$, which is the limit of the set of parallel lines $V_{b}\left(r_{1}\right)(b \in \boldsymbol{R})$. Similarly, $V_{\infty}\left(r_{2}\right)=V(Y-\varepsilon X)$. Since $\left(q_{1}, q_{2}\right)=(Y+\varepsilon X$, $Y-\varepsilon X)=1$, we see that equality holds in (3.8.1), so the hyperbolic fringes in Fig. 1 should approach the limit $V(Y+\varepsilon X) \cup V(Y-\varepsilon X)$ 
as $b \rightarrow \infty$. Of course, this union is the set of two asymptotes of the hyperbolic branchs through $(0,0)$. As $b \rightarrow \infty$, these branches do indeed approach the two lines $Y= \pm \varepsilon X$.

4. Density of rational alternations and moirés at $V_{\infty}$. In this section we extend density results in $\boldsymbol{R}_{X_{1} X_{2}} \backslash V_{\infty}$ noted at the beginning of $\S 3$, to points of $V_{\infty}$. More precisely, let $r_{1}, r_{2}$ be relatively prime in $\boldsymbol{R}\left(X_{1}, X_{2}\right) \backslash \boldsymbol{R}$ (i.e., if $r_{i}=p_{i} / q_{i},\left(p_{i}, q_{i}\right)=1$, then no two of $p_{1}, p_{2}, q_{1}, q_{2}$ share a nonconstant factor). We wish to study the density at points of $V_{\infty}\left(r_{i}\right)$ of $V^{*}\left(r_{i}\right)$, and at points of $V_{\infty}\left(r_{2}-m r_{1}\right)$ of $V^{*}\left(r_{1}\right) \cup V^{*}\left(r_{2}\right)$. That is, let $P$ be a point in $V_{\infty}\left(r_{2}-m r_{1}\right)$, let $\overline{\mathscr{P}}$ be a representative of a real place $\mathscr{P}$ centered at $P$ (as in (4.3)), and let $B(P ; \rho)$ be a disk of radius $\rho$ centered at $P$. The problem is then to evaluate

$$
\lim _{\rho \rightarrow 0} \frac{\text { length }\left[\left(V^{*}\left(r_{1}\right) \cup V^{*}\left(r_{2}\right)\right) \cap C \cap B(P ; \rho)\right]}{\text { length }[C \cap B(P ; \rho)]} .
$$

It turns out that this limit always exists; it is independent of $\mathscr{P}$ if $P \in V_{\infty}\left(r_{2}-m r_{1}\right) \backslash W\left(r_{2}-m r_{1}\right)$, but depends strongly on $\mathscr{P}$ if $P \in$ $W\left(r_{2}-m r_{1}\right)$. We begin by looking at the underlying alternations (corresponding to $m=0$ or $\infty$ ), and then turn to moiré fringes.

In evaluating limits like (4.1), the following result is useful.

Lemma 4.2. Let $\alpha$ be fixed in $\boldsymbol{R} \backslash\{0\} ;$ let $z \in \boldsymbol{R}$. Then

$$
\lim _{z \rightarrow \infty}\left(\frac{(z+1)^{\alpha}-(z+2)^{\alpha}}{z^{\alpha}-(z+1)^{\alpha}}\right)=1
$$

Proof. We may assume without loss of generality that $\alpha \supsetneqq 0$, for

$$
\frac{(z+1)^{-\alpha}-(z+2)^{-\alpha}}{z^{-\alpha}-(z+1)^{-\alpha}}=\left(\frac{z}{z+2}\right)^{\alpha}\left(\frac{(z+1)^{\alpha}-(z+2)^{\alpha}}{z^{\alpha}-(z+1)^{\alpha}}\right) .
$$

Divide numerator and denominator of the quotient in (4.2.1) by $z^{\alpha}$ and set $1 / z=s$; this gives us

$$
\frac{(1+s)^{\alpha}-(1+2 s)^{\alpha}}{1-(1+s)^{\alpha}} \quad(\alpha \supsetneqq 0) .
$$

The binomial series then allows us to rewrite (4.2.2) as

$$
\frac{-\alpha s+s^{2} \cdot[\text { bounded }]}{-\alpha s+s^{2} \cdot[\text { bounded }]} \text {. }
$$

As $z \rightarrow \infty$ (i.e., as $s \rightarrow 0$ ), (4.2.3) approaches 1 . 
Let $r \in \boldsymbol{R}\left(X_{1}, X_{2}\right) \backslash \boldsymbol{R}$. We now consider the density of $V^{*}(r)$ at points of $V_{\infty}(r)$. We consider separately the two cases $P \in V_{\infty}(r) \backslash W(r)$, and $P \in W(r)$.

First, let $P \in W_{\infty}(r) \backslash W(r)$. We wish to show that with respect to any real place $\mathscr{P}$ centered at $P$ and not contained in $V_{\infty}$, the

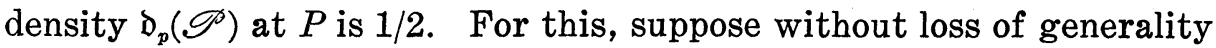
that $P=(0,0)$; then we may write $r=p\left(X_{1}, X_{2}\right) / q\left(X_{1}, X_{2}\right)$, where $p(0,0)=0, q(0,0) \neq 0$. Let $\mathscr{P}$ be a place centered at $(0,0)$, with representative $\overline{\mathscr{P}}$, defined near $(0,0)$ by, say,

$$
\begin{aligned}
& X_{1}=t \\
& X_{2}=f(t) \quad(f \text { real analytic at } t=0) .
\end{aligned}
$$

We may assume without loss of generality that $\boldsymbol{R}_{X_{1}}$ is tangent to $\overline{\mathscr{P}}$. We are interested in the intersections near $(0,0)$ of $\overline{\mathscr{P}}$ with the curves defined by $r=n(n \in \boldsymbol{Z})$. Consider, therefore,

$$
\frac{p\left(t^{\alpha}, f(t)\right)}{q\left(t^{\alpha}, f(t)\right)}=n \quad(|n| \text { large }) .
$$

Since ord $\mathrm{d}_{(0,0)} p=0$ and $\operatorname{ord}_{(0,0)} q \supsetneqq 0$, the order $k$ in $t$ of $q\left(t^{\alpha}, f(t)\right) / p\left(t^{\alpha}, f(t)\right)$ is $k \supsetneqq 1$. Then for $|n|$ sufficiently large, (4.4) has approximate solutions near $t=0$ given by

$$
a t^{k}=\frac{1}{n} \quad(a \in R \backslash(0) \text { independent of } t) .
$$

Therefore we have $t \cong(1 / a n)^{1 / k}$, i.e., $t^{\alpha} \cong(1 / a n)^{\alpha / k}$, or

$$
X_{1} \cong\left(\frac{1}{a n}\right)^{\alpha / k} \text {. }
$$

Since $\boldsymbol{R}_{X_{1}}$ is tangent to $\overline{\mathscr{P}}$ at $(0,0)$, we see that for any large $n$, the distance from $(0,0)$ to any intersection of $r\left(X_{1}, X_{2}\right)=n$ with $\overline{\mathscr{T}}$ is well-approximated by $\left|X_{1}\right|$ in (4.6). (Note that for a fixed, arbitrarily large $n$, there may be zero, one or two such intersections. There are no intersections corresponding to $n$ iff there are two intersections corresponding to $-n$.) But (4.6) together with Lemma 4.2 shows that the distance measured along $\overline{\mathscr{P}}$ between successive points of intersection of $\overline{\mathscr{P}}$ with the curves $r=n$, become more and more nearly equal as $|n| \rightarrow \infty$. From Definition 2.3, one easily sees that in this case the density $\mathfrak{D}_{P}(\mathscr{P})$ of $V^{*}(r)$ along $\mathscr{P}$ is $1 / 2$.

We now look at the case when $P \in W(r)$. Let $\overline{\mathscr{P}}$ be as above. Now in contrast to the situation of $P \in V_{\infty}(r) \backslash W(r)$, there may be either infinitely many or only finitely many $n \in Z$ such that the curve $V_{n}=V_{n}(r)$ intersects $\overline{\mathscr{P}}$ near $(0,0)$. We consider these two possibilities

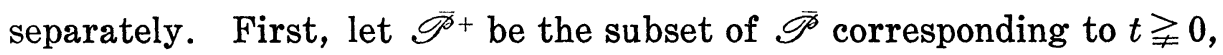


and let $\overline{\mathscr{P}}^{-}$be the subset of $\overline{\mathscr{P}}$ corresponding to $t \supseteqq 0$.

We now look at the "infinite" case. In this case

There are both infinitely many points of $V_{n} \cap \overline{\mathscr{P}}^{+}$near $(0,0)$, and infinitely many points of $V_{n} \cap \overline{\mathscr{P}}^{-}$near $(0,0)$.

(4.7) easily follows from this fact: if $q\left(t^{\alpha}, f(t)\right) / p\left(t^{\alpha}, f(t)\right)$ is unbounded as $t \rightarrow 0$ from one side, then it is unbounded as $t \rightarrow 0$ from the other side. This fact also implies that $\operatorname{ord}_{0}\left(p\left(t^{\alpha}, f(t)\right)\right) \neq \operatorname{ord}_{0}\left(q\left(t^{\alpha}, f(t)\right)\right)$. Then $k$ in (4.6) is replaced by $\operatorname{ord}_{0}\left(q\left(t^{\alpha}, f(t)\right)\right)-\operatorname{ord}_{0}\left(p\left(t^{\alpha}, f(t)\right)\right)$, and an argument like that in the case $P \in V_{\infty}(r) \backslash W(r)$ (using Lemma 4.2 and (4.7)) shows that on each of $\overline{\mathscr{P}}^{+}$and $\overline{\mathscr{P}}^{-}$, the density $\mathfrak{D}_{P}(\mathscr{P})$ is $1 / 2$; hence in this case the density $\mathfrak{D}_{P}(\mathscr{P})$ of $V^{*}(r)$ along $\mathscr{P}$ is $1 / 2$.

We now consider the other case, when there are not infinitely many $n \in Z$ such that $V_{n}(r)$ intersects $\mathscr{P}$ near $(0,0)$. Then for a sufficiently small neighborhood $U$ about $(0,0), \mathscr{\mathscr { P }}^{+} \cap U$ is either contained in $V^{*}(r)$, or else is contained in $\boldsymbol{R}_{x_{1} x_{2}} \backslash V^{*}(r)$. In the first instance, the density of $V^{*}(r) \cap \overline{\mathscr{P}}^{+}$in $\overline{\mathscr{P}}^{+}$is 1 and in the second, it is 0 ; similarly for $\mathscr{P}^{-}$. Thüs in this case

the density $\mathfrak{D}_{P}(\mathscr{P})$ of $V^{*}(r) \cap \overline{\mathscr{P}}$ along $\overline{\mathscr{P}}$ is $1,1 / 2$ or 0 depending on whether both, exactly one, or neither $\mathscr{P}^{+} \cap U$, $\overrightarrow{\mathscr{P}} \cap U$ is contained in $V^{*}(r)$.

In the above "finite" case, all three possibilities can occur, as the following example shows.

EXAMPle 4.8. Let $r=X_{2} / X_{1} \in \boldsymbol{R}\left(X_{1}, X_{2}\right) \backslash \boldsymbol{R}$; let $P=(0,0)$.

4.8.1. If $\mathscr{P}$ is defined by

$$
\left\{\begin{array}{l}
X_{1}=2 t \\
X_{2}=t
\end{array}\right.
$$

then $\overline{\mathscr{P}} \cong V^{*}\left(X_{2} / X_{1}\right)$, so $\grave{D}_{P}(\mathscr{P})=1$.

4.8.2. If $\mathscr{P}$ is defined by

$$
\left\{\begin{array}{l}
X_{1}=-2 t \\
X_{2}=t
\end{array}\right.
$$

then $\ddot{\mathscr{P}} \subseteq \boldsymbol{R}_{X_{1} X_{2}} \backslash V^{*}\left(X_{2} / X_{1}\right)$, so $\mathfrak{D}_{P}(\mathscr{P})=0$.

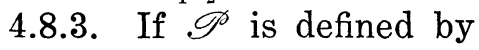

$$
\left\{\begin{array}{l}
X_{1}=t^{2} \\
X_{2}=t^{3}
\end{array}\right.
$$

then for sufficiently small $\overline{\mathscr{P}}^{+}, \overline{\mathscr{P}}^{+} \subseteq V^{*}\left(X_{2} / X_{1}\right)$, and $\left.\overline{\mathscr{P}}^{-} \leqq \boldsymbol{R}_{X_{1} X_{2}}\right)$ 
$V^{*}\left(X_{2} / X_{1}\right)$. Thus in this case $\grave{D}_{P}(\mathscr{P})=1 / 2$.

We now consider densities of moirés at points $P$ of $V_{\infty}\left(r_{2}-m r_{1}\right)$. Let $r_{1}, r_{2} \in \boldsymbol{R}\left(X_{1}, X_{2}\right) \backslash \boldsymbol{R},\left(r_{1}, r_{2}\right)=1$, and let $m \in \boldsymbol{R}$. We look separately at the two cases $P \in V_{\infty}\left(r_{2}-m r_{1}\right) \backslash W\left(r_{2}-m r_{1}\right)$, and $P \in W\left(r_{2}-m r_{1}\right)$.

First, suppose $P \in V_{\infty}\left(r_{2}-m r_{1}\right) \backslash W\left(r_{2}-m r_{1}\right)$. Let $\mathscr{P}$ be any real place centered at $P$ and not contained in $V_{\infty}\left(r_{2}-m r_{1}\right)$; let $\overline{\mathscr{P}}$ be a representative of $\mathscr{P}$. As a function of $b$, the density $\mathfrak{D}\left(V_{b}\right)$ of $\left(V^{*}\left(r_{1}\right) \cup\right.$ $\left.V^{*}\left(r_{2}\right)\right) \cap V_{b}(b \in \boldsymbol{R})$ in $V_{b}$ is either $3 / 4$, or else it oscillates about $3 / 4$, with formulas analogous to those in Theorem 2.7 (cf. §3). Using Lemma 4.2 and the piecewise linearity with respect to $b$ of $\mathfrak{D}\left(V_{b}\right)$, we see that for any $\varepsilon>0$, there exists a sufficiently large positive integer $n_{0}$ so that for any $n\left(|n| \geqq n_{0}\right)$, the density of $V^{*}\left(r_{1}\right) \cup V^{*}\left(r_{2}\right)$ measured over the part of $\overline{\mathscr{P}}$ corresponding to $1 /(n+2) \leqq t \leqq 1 / n$ is within $\varepsilon$ of $3 / 4$. Thus for any $\varepsilon>0$, the density of $V^{*}\left(r_{1}\right) \cup V^{*}\left(r_{2}\right)$ within the part of $\overline{\mathscr{P}}$ corresponding to all sufficiently large $|t|$, is within $\varepsilon$ of $3 / 4$. Therefore at $P$ itself $\left(P \in V_{\infty}\left(r_{2}-m r_{1}\right) \backslash W\left(r_{2}-m r_{1}\right)\right)$, the density of $V^{*}\left(r_{1}\right) \cup V^{*}\left(r_{2}\right)$ with respect to $\mathscr{P}$, is $3 / 4$.

Now suppose $P \in W_{\infty}\left(r_{2}-m r_{1}\right)$. Let $V_{b}=V_{b}\left(r_{2}-m r_{1}\right)$ be a fixed fringe curve through $P$. Then for any $\varepsilon>0$, there exists a sufficiently small neighborhood $U_{P}$ about $P$ such that the density of $\left(V^{*}\left(r_{1}\right) \cup\right.$ $\left.V^{*}\left(r_{2}\right)\right) \cap V_{b}$ measured along $V_{b} \cap U_{P}$, is within $\varepsilon$ of $D\left(V_{b}\right)$. This is because for any $Q \in V_{b}$ sufficiently close to $P(Q \neq P)$, the set of points of $\left(V^{*}\left(r_{1}\right) \cup V^{*}\left(r_{2}\right)\right) \cap V_{b}$ near $Q$ has a repeating pattern which is arbitrarily well-approximated by the pattern $R \cap V_{b}$ about any fixed point $\neq P$, where $R$ is a sufficiently high $m$-refinement of $V^{*}\left(r_{1}\right) \cup V^{*}\left(r_{2}\right)$. Thus the density at $P \in W\left(r_{0}-m r_{1}\right)$ along $V_{b}\left(r_{2}-m r_{1}\right)$ $(b \in R)$ is just $\mathfrak{D}\left(V_{b}\right)$.

\section{REFERENCES}

1. K. Kendig, Tangent cones to real analytic varieties, Indiana University Math. J., 22, No. 4 (1972), 379-391.

2. - Moiré Phenomena in Algebraic Geometry: Polynomial Alternations in $\boldsymbol{R}^{n}$. Pacific J. Math., 88 (1980).

3. G. Oster, Moiré Optics: A Bibliography, J. Optical Soc. Amer., 55 (1965), 1329.

4. V. Ronchi, Sur la Nature Interferentielle des Franges d'ombre dans l'essai des Systems Optiques, Revue Opt., 5, No. 11 (1926), 431-437.

5. P. Theocaris, Moiré Fringes in Strain Analysis, London: Permagon Press, 1969.

Received May 9, 1979.

Cieveland State University

Cieveland, OH 44115 


\section{PACIFIC JOURNAL OF MATHEMATICS}

\section{EDITORS}

DONALD BABBITT (Managing Editor)

University of Galifornia

Los Angeles, California 90024

HUGO RossI

University of Utah

Salt Lake City, UT 84112

C. C. MOORE AND ANDREW OGG

University of California

Berkeley, CA 94720
J. DUGUNDJI

Department of Mathematics University of Southern California Los Angeles, California 90007

R. FINN AND J. MILGRAM Stanford University

Stanford, California 94305

ASSOCIATE EDITORS

E. F. BECKENBACH

B. H. NeumanN

F. WOLF

K. YosHidA

\section{SUPPORTING INSTITUTIONS}

UNIVERSITY OF BRITISH COLUMBIA UNIVERSITY OF SOUTHERN CALIFONIA CALIFORNIA INSTITUTE OF TECHNOLOGY UNIVERSITY OF CALIFORNIA MONTANA STATE UNIVERSITY STANFORD UNIVERSITY UNIVERSITY OF HAWAII UNIVERSITY OF NEVADA, RENO UNIVERSITY OF TOKYO U'NIVERSITY OF UTAH NEW MEXICO STATE UNIVERSITY WASHINGTON STATE UNIVERSITY OREGON STATE UNIVERSITY UNIVERSITY OF OREGON UNIVERSITY OF WASHINGTON 


\section{Pacific Journal of Mathematics}

\section{Vol. 90, No. $1 \quad$ September, 1980}

Shashi Prabha Arya and M. K. Singal, On the locally countable sum

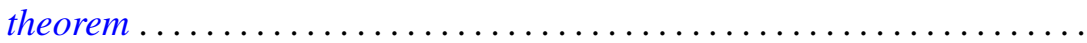

John Theodore Baldwin and David William Kueker, Ramsey quantifiers and

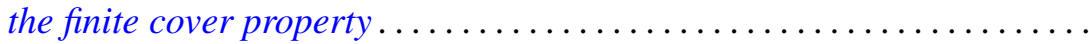

Richard Body and Roy Rene Douglas, Unique factorization of rational

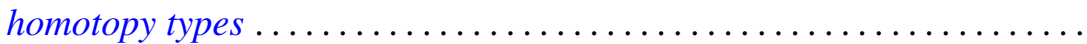

Ethan Bolker and Ben G. Roth, When is a bipartite graph a rigid

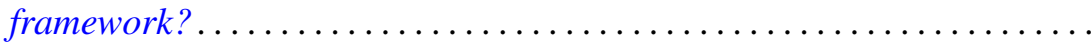

Alicia B. Winslow, Continua in the Stone-Čech remainder of $R^{2} \ldots \ldots \ldots$

Richard D. Carmichael and Elmer Kinji Hayashi, Analytic functions in tubes which are representable by Fourier-Laplace integrals ..............

Stephen D. Cohen, The Galois group of a polynomial with two indeterminate coefficients ..............................

Russell Allan Johnson, Strong liftings commuting with minimal distal

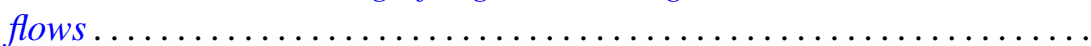

Elgin Harold Johnston, The boundary modulus of continuity of harmonic

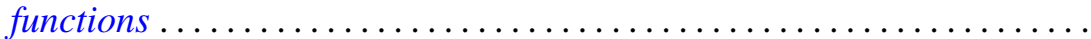

Akio Kawauchi and Takao Matumoto, An estimate of infinite cyclic

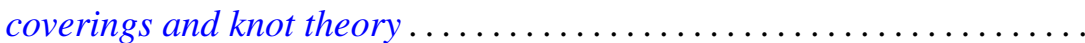

Keith Milo Kendig, Moiré phenomena in algebraic geometry: rational alternations in $\mathbf{R}^{2}$...

Roger T. Lewis and Lynne C. Wright, Comparison and oscillation criteria for selfadjoint vector-matrix differential equations .

Teck Cheong Lim, Asymptotic centers and nonexpansive mappings in conjugate Banach spaces .......................

David John Lutzer and Robert Allen McCoy, Category in function spaces.

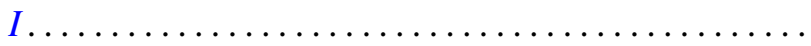

Richard A. Mollin, Induced p-elements in the Schur group ...

Jonathan Simon, Wirtinger approximations and the knot groups of $F^{n}$ in $S^{n+2}$

Robert L. Snider, The zero divisor conjecture for some solvable groups...

H. M. (Hari Mohan) Srivastava, A note on the Konhauser sets of biorthogonal polynomials suggested by the Laguerre polynomials...

Nicholas Th. Varopoulos, A probabilistic proof of the Garnett-Jones theorem on BMO.

Frank Arvey Wattenberg, $[0, \infty]$-valued, translation invariant measures on $N$ and the Dedekind completion of ${ }^{*} R \ldots \ldots \ldots \ldots . .$. 